\title{
Multi-Objective Optimization of AWJM of Lead Tin Alloy by GRA
}

\author{
M. Dev Anand, K.S. Jai Aultrin, G. Ramanan, R.R. Neela Rajan
}

\begin{abstract}
Last decades have witnessed a rapid growth in the development of harder, difficult and complexity to machine metals and alloys. AWJM is one of the most freshly built up non traditional machining in processing various types of hard-to-cut materials nowadays. It is an economical method for heat sensitive materials that cannot be machined by processes that produce heat while machining. Machining parameters play the lead position in bringing out the machine economics and machining quality. This research examines the persuading and parametric optimization of five parameters of the process for Abrasive water jet machining of Lead Tin alloy involving grey relation analysis. Depending upon RSM various sets of research have been performed on this element by changeable five different parameters on MRR and SR. ANOVA has been carried out to recognize the noteworthy parameters which affect toughness of abrasive waterjet machining process. The consequence of the experiments for best possible setting proves that there is extensive development in the process. The main objective of grey relational analysis is to translate the multi response variable in to a single response grade.
\end{abstract}

Keywords--- Abrasive Water Jet Machining, Grey Relational Analysis, Response Surface Methodology, Material Removal Rate, Surface Roughness.

\section{NOMENCLATURE}

\begin{tabular}{|c|l|l|c|}
\hline Sl. No. & \multicolumn{1}{|c|}{ Factors } & Symbol & Unit \\
\hline $\mathbf{1 .}$ & Water Pressure & $\mathrm{P}$ & Bar \\
\hline $\mathbf{2 .}$ & Abrasive Flow Rate & $\mathrm{m}_{\mathrm{f}}$ & $\mathrm{Kg} / \mathrm{min}$ \\
\hline $\mathbf{3 .}$ & Orifice Diameter & $\mathrm{d}_{\mathrm{o}}$ & $\mathrm{mm}$ \\
\hline $\mathbf{4 .}$ & Focusing Nozzle & $\mathrm{d}_{\mathrm{f}}$ & $\mathrm{mm}$ \\
\hline $\mathbf{5 .}$ & Stand Off Distance & $\mathrm{S}$ & $\mathrm{mm}$ \\
\hline $\mathbf{6 .}$ & Material Removal Rate & MRR & $\mathrm{mm}^{3} / \mathrm{min}$ \\
\hline $\mathbf{7 .}$ & Surface Roughness & $\mathrm{SR}$ & $\mu \mathrm{m}$ \\
\hline
\end{tabular}

\section{INTRODUCTION AND LITERATURE SURVEY}

AWJC, a known and the recently built up method is suitable for machining of brittle materials namely stones, glass and ceramics as well as for ferrous, composite materials and non-ferrous materials.

From the literature review of Momber and Kovacevic (1992) consider this technology is not as much of responsive to properties of material since it has not created chatter,

Revised Manuscript Received on July 10, 2019.

M. Dev Anand, Professor and Director Research, Department of Mechanical Engineering, Noorul Islam Centre for Higher Education, Kumaracoil, Tamil Nadu, India.

K.S. Jai Aultrin, Associate Professor, Department of Mechanical Engineering, Noorul Islam Centre for Higher Education, Kumaracoil, Tamil Nadu, India.

G. Ramanan, Assistant Professor, Department of Aerospace Engineering, ACS Collge of Engineering, Banglore, Karnataka, India.

R.R. Neela Rajan, Assistant Professor, Department of Aeronautical Engineering, Noorul Islam Centre for Higher Education, Kumaracoil, Tamil Nadu, India. without heat effects, inflict stresses of minimum level over the workpiece, and possess greater usefulness in machining and much flexibility [1]. Hashish M. (1989) reviewed that in this technique, a rivulet of little particles that are abrasive is pioneered in the waterjet and this combination abrasive water jet is later intended to impact on the working area to cut it [2]. In AWJC, less effort was taken for modeling and optimizing the parameters for the process. The move engaged along this track comprises regression modeling, fuzzy logics, Analysis Of Variance (ANOVA), artificial neural networks and Design of Experiments (DOE). Few of these research offer increase to numerous numerical equations brought out for forecasting the output parameters [3].

R. Ravi Kumar, M. Santhi \& R. Jeyapaul \& P. Asokan in their paper [4], flow rate, voltage, current and gap are measured as metal removal rate, machining parameters and surface roughness were the targets. Later on application of grey relational analysis, they calculated the grey grade to represent multi-objective model. ANN model and multiple regression model were built up to plot the association among parameters of the process and targets known as grade. D. Chakradhar, A. Venu Gopal in their paper [5], examines the process parameters optimization and effect for Electrochemical machining of EN-31 steel involving grey relation analysis. C. L. Lin in his article [6], deal with a technique depending on the Taguchi method along grey relational analysis for optimizing turning operations along the multiple performance characteristics. From the literature review of R.M. Arunachalam and N. Natarajan [7], optimization of micro-EDM along numerous concert distinctiveness involving Grey Relational Analysis and Taguchi Method has been done.

From J. L. Lin, T. C. Ko and C. L. Lin paper [8], the utilize the grey relational analysis depending upon an orthogonal array and fuzzy-based Taguchi method to optimize the multi-response process is accounted in EDM process.

In this paper, optimization of abrasive water jet machining process of Lead Tin alloy by grey relational analysis has been presented.

\section{EXPERIMENTAL WORK}

\section{Material}

The well known American element named Lead Tin alloy, exist in numerous appearances namely tube, ribbon, ingot, foil, wire, bar, rod and pipe. 


\section{Multi-Objective Optimization Of Awjm Of Lead Tin Alloy By Gra}

Significant feature in choosing Lead Tin alloy is their high strength to weight ratio, their resistance against corrosion by numerous chemicals, their high thermal and electrical conductivity, reflectivity and appearance, non toxicity and their easiness of formability and machinability and non magnetic properties. Few applications of Lead Tin alloy include aerospace maintenance, brake components, bicycle frames, Marine fittings, transport, valves couplings etc. It is also applied in paint removal, surgery, peening, drilling turning etc. Good surface finish exists and could also be anodized. Exclusive corrosion resistance to atmospheric conditions exists. Density of it is $11.035 \mathrm{~g} / \mathrm{cm} 3$ and its Modulus of Elasticity E $=80 \mathrm{GPa}$. Lead Tin alloy plate dimension utilized in this research is $150 \mathrm{~mm} \times 50 \mathrm{~mm}$ x 50mm. shown in Figure 1.



Figure 1: Lead Tin Alloy helpful to model and analyse troubles. In the present study five process parameters are chosen and assorted at three levels that have been exhibited in Table 1 and the frequently utilized parameters that seem to be constant of AWJM is illustrated in Table 2. Table 3 depicts the steps concerned in the RSM optimization process.

Table 1: Levels of Parameters Used in Experiment

\begin{tabular}{|l|c|c|c|c|c|}
\hline Levels & $\begin{array}{c}\text { Water } \\
\text { Pressu } \\
\text { re }(\mathbf{P}) \\
\text { Bar }\end{array}$ & $\begin{array}{c}\text { Abrasi } \\
\text { ve } \\
\text { Flow } \\
\text { Rate } \\
\left(\mathbf{m}_{\mathbf{f}}\right) \\
\mathbf{K g} / \mathbf{m}^{3}\end{array}$ & $\begin{array}{c}\text { Orifice } \\
\text { Diamet } \\
\text { er }\left(\mathbf{d}_{\mathbf{o}}\right) \\
\mathbf{m m}\end{array}$ & $\begin{array}{c}\text { Focusi } \\
\mathbf{n g} \\
\text { Nozzle } \\
\text { Diamet } \\
\mathbf{e r}\left(\mathbf{d}_{\mathbf{f}}\right) \\
\mathbf{m m}\end{array}$ & $\begin{array}{c}\text { Stand } \\
\text { Off } \\
\text { Distan } \\
\text { ce (s) } \\
\mathbf{m m}\end{array}$ \\
\hline Low & 3400 & 0.4 & 0.3 & 0.9 & 1 \\
\hline Intermedi & 3600 & 0.55 & 0.33 & 0.99 & 2 \\
\hline High & 3800 & 0.7 & 0.35 & 1.05 & 3 \\
\hline
\end{tabular}

Table 2: Constant Parameters

\begin{tabular}{|c|l|l|}
\hline Sl. & \multicolumn{1}{|c|}{ Parameters } & \multicolumn{1}{|c|}{ Type / Value } \\
\hline $\mathbf{1 .}$ & Jet Impact Angle & Neutral nozzle position $\left(90^{\circ}\right)$ \\
\hline $\mathbf{2 .}$ & Nozzle Length & $76.2 \mathrm{~mm}$ \\
\hline $\mathbf{3 .}$ & Size of Abrasive & $80 \mathrm{mesh}$ garnet \\
\hline $\mathbf{4 .}$ & Type of Abrasive & Hard rock \\
\hline $\mathbf{5 .}$ & Diameter of & $0.18 \mathrm{~mm}$ \\
\hline $\mathbf{6 .}$ & Density of Garnet & $4100 \mathrm{~kg} / \mathrm{m}^{3}$ \\
\hline $\mathbf{7 .}$ & $\begin{array}{l}\text { Composition of } \\
\text { garnet }\end{array}$ & $\begin{array}{l}36 \% \mathrm{FeO}, \quad 33 \% \quad \mathrm{SiO}_{2}, \quad 20 \% \\
\mathrm{Al}_{2} \mathrm{O}_{3}, \quad 4 \% \mathrm{MgO}, 3 \% \quad \mathrm{TiO}_{2},\end{array}$ \\
\hline
\end{tabular}

Involving 46 experiments from a Box-Behnken design table, in response surface design with has been chosen and has been shown in Figure 2. The parameters and levels have been chosen as per the review of few journals which was accepted on AWJC on titanium [9], Mildsteel [10] Copper [11] and epoxy composite laminate [12].

\section{Response Surface Methodology}

Response Surface Methodology is a gathering of numerical and statistical techniques which seems to be








Figure 2: Selection of Box-Behnken Design and Selection of No of Factors

Table 3: Steps Involved in RSM Optimization Process

\begin{tabular}{|c|c|}
\hline Steps & Process \\
\hline First & Response surface Methodology Design \\
\hline Second & Experimental Datas of MRR and SR \\
\hline Third & Mathematical Modeling for MRR and SR \\
\hline Fourth & Grey Relational Analysis \\
\hline Fifth & Analysis of Variance \\
\hline Sixth & Determination of Optimal Design \\
\hline Seventh & Confirmation Test \\
\hline
\end{tabular}

Data Collection and Experimentation

For all experiments the parameters involved in cutting have been accustomed to the pre-defined levels. Forty six experiment have been performed here in Lead Tin alloy according to Box-Behnken design preferred. Equipment or machine utilized for cutting Lead Tin alloy, which is the
American element, was the AWJC machine has been prepared with KMT pressure pump which is ultrahigh holding the designed pressure of 4000bar, abrasive hopper which is of gravity feed type, a feeder system of abrasive, a valve controlled pneumatically and a work piece table. In the course of the controller used which is permanent in the control stand, SOD has been attuned for numerous experiments. The abrasive water jet scheme programmed using numerical control code has been utilized for adjusting the transverse speed and controlling the abrasives supplement. After the water is pumped at very high pressures resulting in greater velocity of water jet of 1000 $\mathrm{m} / \mathrm{s}$ as it egress off the nozzle cuts to the desired size and shape of material. The KMT abrasive water jet cutting machine is shown in Figure 3 with its mixing chamber.


Figure 3: Experimental Setup of AWJM with Mixing Chamber




Multi-Objective Optimization Of Awjm Of Lead Tin Alloy By Gra

Table 4: Scheduling Matrix of the Experiments with the Optimal Model Data

\begin{tabular}{|c|c|c|c|c|c|c|c|}
\hline Sl. No. & $\begin{array}{l}\text { Pressure } \\
\text { (Bar) }\end{array}$ & $\begin{array}{c}\text { Abrasive Flow } \\
\text { Rate } \\
\text { (Kg/min) }\end{array}$ & $\begin{array}{l}\text { Orifice Diameter } \\
\quad(\mathbf{m m})\end{array}$ & $\begin{array}{c}\text { Focusing Tube } \\
\text { Diameter } \\
(\mathrm{mm})\end{array}$ & $\begin{array}{l}\text { Stand Off Distance } \\
(\mathbf{m m})\end{array}$ & $\underset{\mathrm{mm}^{3} / \mathrm{min}}{\mathrm{MRR}}$ & $\begin{array}{c}\text { SR } \\
(\mu \mathrm{m})\end{array}$ \\
\hline 1. & 3400 & 0.55 & 0.33 & 0.99 & 3 & 1709 & 2.45 \\
\hline 2. & 3600 & 0.55 & 0.33 & 0.9 & 1 & 2014.86 & 1.415 \\
\hline 3. & 3600 & 0.55 & 0.3 & 1.05 & 2 & 1970.09 & 1.624 \\
\hline 4. & 3600 & 0.55 & 0.33 & 0.9 & 3 & 1916.85 & 2.2 \\
\hline 5. & 3800 & 0.55 & 0.33 & 0.9 & 2 & 2182.26 & 0.788 \\
\hline 6. & 3600 & 0.55 & 0.33 & 0.99 & 2 & 1997.84 & 1.609 \\
\hline 7. & 3400 & 0.4 & 0.33 & 0.99 & 2 & 1688.65 & 2.109 \\
\hline 8. & 3600 & 0.7 & 0.35 & 0.99 & 2 & 1997.84 & 1.52 \\
\hline 9. & 3800 & 0.55 & 0.33 & 0.99 & 3 & 2085.98 & 1.201 \\
\hline 10. & 3800 & 0.55 & 0.3 & 0.99 & 2 & 2149.19 & 0.801 \\
\hline 11. & 3600 & 0.55 & 0.33 & 0.99 & 3 & 1896.34 & 2.1 \\
\hline 12. & 3400 & 0.55 & 0.33 & 1.05 & 2 & 1746.88 & 1.905 \\
\hline 13. & 3600 & 0.4 & 0.33 & 0.99 & 1 & 1943.11 & 1.887 \\
\hline 14. & 3600 & 0.55 & 0.33 & 0.99 & 2 & 2009.16 & 1.571 \\
\hline 15. & 3600 & 0.55 & 0.35 & 0.9 & 2 & 1948.44 & 1.53 \\
\hline 16. & 3600 & 0.55 & 0.3 & 0.9 & 2 & 2003.48 & 1.709 \\
\hline 17. & 3400 & 0.55 & 0.33 & 0.9 & 2 & 1751.19 & 1.9 \\
\hline 18. & 3600 & 0.55 & 0.33 & 0.99 & 2 & 2003.48 & 1.566 \\
\hline 19. & 3600 & 0.4 & 0.3 & 0.99 & 2 & 1842.16 & 1.91 \\
\hline 20. & 3400 & 0.55 & 0.35 & 0.99 & 2 & 1751.19 & 1.899 \\
\hline 21. & 3800 & 0.4 & 0.33 & 0.99 & 2 & 2136.25 & 1.211 \\
\hline 22. & 3600 & 0.7 & 0.33 & 0.99 & 3 & 1891.29 & 1.999 \\
\hline 23. & 3600 & 0.7 & 0.33 & 0.99 & 1 & 2055.75 & 1.431 \\
\hline 24. & 3600 & 0.4 & 0.35 & 0.99 & 2 & 1866.4 & 2.013 \\
\hline 25. & 3600 & 0.4 & 0.33 & 0.9 & 2 & 1842.16 & 1.945 \\
\hline 26. & 3600 & 0.55 & 0.35 & 0.99 & 3 & 1916.85 & 2.008 \\
\hline 27. & 3600 & 0.7 & 0.33 & 0.9 & 2 & 1970.09 & 1.5 \\
\hline 28. & 3400 & 0.55 & 0.33 & 0.99 & 1 & 1800.08 & 1.789 \\
\hline 29. & 3600 & 0.7 & 0.3 & 0.99 & 2 & 1937.8 & 1.699 \\
\hline 30. & 3600 & 0.55 & 0.33 & 1.05 & 1 & 2049.81 & 1.707 \\
\hline 31. & 3600 & 0.55 & 0.3 & 0.99 & 1 & 2009.16 & 1.5 \\
\hline 32. & 3800 & 0.7 & 0.33 & 0.99 & 2 & 2142.69 & 0.62 \\
\hline 33. & 3600 & 0.4 & 0.33 & 1.05 & 2 & 1866.4 & 1.934 \\
\hline 34. & 3600 & 0.55 & 0.3 & 0.99 & 3 & 1916.84 & 2.309 \\
\hline 35. & 3600 & 0.55 & 0.33 & 0.99 & 2 & 2014.86 & 1.597 \\
\hline 36. & 3800 & 0.55 & 0.33 & 1.05 & 2 & 2162.3 & 0.8 \\
\hline 37. & 3400 & 0.7 & 0.33 & 0.99 & 2 & 1800.08 & 1.9 \\
\hline 38. & 3600 & 0.55 & 0.35 & 1.05 & 2 & 2020.6 & 1.704 \\
\hline 39. & 3400 & 0.55 & 0.3 & 0.99 & 2 & 1768.66 & 2.102 \\
\hline 40. & 3600 & 0.4 & 0.33 & 0.99 & 3 & 1842.16 & 2.345 \\
\hline 41. & 3600 & 0.55 & 0.33 & 0.99 & 2 & 2020.6 & 1.64 \\
\hline 42. & 3600 & 0.55 & 0.35 & 0.99 & 1 & 2079.86 & 1.634 \\
\hline 43. & 3800 & 0.55 & 0.35 & 0.99 & 2 & 2162.3 & 0.881 \\
\hline 44. & 3600 & 0.7 & 0.33 & 1.05 & 2 & 1970.09 & 1.539 \\
\hline 45. & 3600 & 0.55 & 0.33 & 1.05 & 3 & 1922.04 & 1.997 \\
\hline 46. & 3800 & 0.55 & 0.33 & 0.99 & 1 & 2223.3 & 0.8 \\
\hline
\end{tabular}


In Design of Experiments, depending upon Box-behnken design and Response surface methodology for five factors along 46 experiments has been chosen and performed investigational and machining time is got for every experiment is shown in Table 4. The MRR is premeditated experimentally involving the following formula;

MRR $=($ Initial Weight - Final Weight $) /$ Machining

Time $=\left(\mathrm{m}_{\mathrm{f}}-\mathrm{m}_{\mathrm{i}}\right) / \mathrm{t}$

Where,

$\mathrm{m}_{\mathrm{f}}=$ material mass after machining

$\mathrm{m}_{\mathrm{i}}=$ material mass before machining

$\mathrm{t}=$ Machining Time

The surface roughness for the machined Lead Tin alloy is measured utilizing Portable surface roughness tester in

National College of Engineering, Tamil Nadu, India, is shown in Figure 4.



Figure 4: Surface Roughness Tester

\section{GREY RELATIONAL ANALYSIS \& RESULTS}

Grey relational analysis is applied in finding out the best choice of machining parameters for any machining process.

According to the Response surface methodology design 46 sets of experiments are chosen for the experimentation and MRR and SR is found out. The higher-the-better functioning for MRR might influence the performance due to that the SR may maintain lower-the-better uniqueness. Therefore, multi-response optimization distinctiveness were known to be complex. In this part, the usage of response surface methodology with the methodology for optimization which is referred to be Grey relational analysis mainly targeting multi-response optimization has been conversed. The Table 5 shows the steps takes place in optimization of the process using Grey relational analysis.

Table 5: Steps in Optimization Using Grey Relational Analysis

\begin{tabular}{|l|l|}
\hline Steps & Process \\
\hline First & Prepossessing the data results of MRR and SR. \\
\hline Second & $\begin{array}{l}\text { Grey relational generation is performed and Grey } \\
\text { relational coefficient is premeditated. }\end{array}$ \\
\hline Third & $\begin{array}{l}\text { Using the GRC values GRG is calculated by averaging } \\
\text { the Grey relational coefficient. }\end{array}$ \\
\hline Fourth & $\begin{array}{l}\text { ANOVA is done to determine the parameter that } \\
\text { drastically influences the process and design which is } \\
\text { optimal is identified. }\end{array}$ \\
\hline Fifth & Confirmation experiment is done by setting the optimal \\
\hline
\end{tabular}

process parameters to provide the optimal design confirmation.

The following formulas are applied to find the Normalizing the experimental results, Grey relational coefficient and Grey relational grade.

(a) For higher the better quality and lesser the better quality, Normalizing the experimental outcomes are given by,

$$
\begin{aligned}
& \mathrm{X}_{\mathrm{i}}(\mathrm{k})=\left(\mathrm{y}_{\mathrm{i}}(\mathrm{k})-\min \mathrm{y}_{\mathrm{i}}(\mathrm{k})\right) /\left(\max \mathrm{y}_{\mathrm{i}}(\mathrm{k})-\min \mathrm{y}_{\mathrm{i}}(\mathrm{k})\right) \\
& \mathrm{X}_{\mathrm{i}}(\mathrm{k})=\left(\max \mathrm{y}_{\mathrm{i}}(\mathrm{k})-\mathrm{y}_{\mathrm{i}}(\mathrm{k})\right) /\left(\max \mathrm{y}_{\mathrm{i}}(\mathrm{k})-\min \mathrm{y}_{\mathrm{i}}(\mathrm{k})\right)
\end{aligned}
$$

Where,

$\mathrm{X}_{\mathrm{i}}(\mathrm{k})$ is the value after grey relation generation

$\min \mathrm{y}_{\mathrm{i}}(\mathrm{k})$ is the minimum value of $\mathrm{y}_{\mathrm{i}}(\mathrm{k})$ for the $\mathrm{k}^{\text {th }}$ response $\max \mathrm{y}_{\mathrm{i}}(\mathrm{k})$ is the biggest value of $\mathrm{y}_{\mathrm{i}}(\mathrm{k})$ for the $\mathrm{k}^{\text {th }}$ response.

(b) Calculating the grey relational coefficient from the normalized values yield.

$$
\begin{aligned}
& \gamma\left(\mathrm{x}_{\mathrm{o}}(\mathrm{k}), \mathrm{x}_{\mathrm{i}}(\mathrm{k})\right)=(\Delta \min +\zeta \Delta \max ) /\left(\Delta_{\mathrm{oj}}(\mathrm{k})+\zeta \Delta \max \right)(3) \\
& \text { Where, }
\end{aligned}
$$

$\mathrm{j}=1,2 \ldots \mathrm{n} ; \mathrm{k}=1,2 \ldots \mathrm{m}, \mathrm{n}$ is experimental data number and $\mathrm{m}$ is responses number.

$\mathrm{X}_{\mathrm{o}}(\mathrm{k})$ is reference sequence $\left(\mathrm{x}_{\mathrm{o}}(\mathrm{k})=1, \mathrm{k}=1,2 \ldots \mathrm{m}\right) ; \mathrm{x}_{\mathrm{j}}(\mathrm{k})$ is specific comparison sequence.

$\Delta_{\mathrm{oj}}=\left\|\mathrm{x}_{\mathrm{o}}(\mathrm{k})-\mathrm{x}_{\mathrm{j}}(\mathrm{k})\right\|=$ The absolute value of the difference between $x_{o}(k)$ and $x_{j}(k)$.

$\Delta \min =\operatorname{minmin}\left\|x_{o}(k)-x_{j}(k)\right\|$ is the smallest value of $x_{j}(k)$.

$\Delta \max =\operatorname{maxmax}\left\|\mathrm{x}_{\mathrm{o}}(\mathrm{k})-\mathrm{x}_{\mathrm{j}}(\mathrm{k})\right\|$ is the largest value of $\mathrm{x}_{\mathrm{j}}(\mathrm{k})$.

$\zeta$ is the distinguishing coefficient in the range $0 \leq \zeta \leq 1$ (the value may adjusted based on the practical needs of the system).

(c) Calculating the grey relational grade is done by averaging the grey relational coefficient which contributes:

$$
\gamma \mathrm{j}=\frac{1}{\mathrm{k}} \sum \gamma \mathrm{ij} \text {-------- (4) }
$$

$\gamma \mathrm{j}$ is $\mathrm{jth}$ grey relational grade and performance numbers characteristics is $\mathrm{k}$. Normalized data results for SR and MRR is depicted in Table 6, the grey relational co-efficient from normalized values for MRR and SR is illustrated in Table 7 and Table 8 depicts the authority of parameters involved in process of Grey Relational Grade for Lead Tin alloy. 
Table 6: Normalized Data Results

\begin{tabular}{|c|c|c|c|c|}
\hline Sl. No. & Material Removal Rate & Surface Roughness & $\begin{array}{l}\text { Normalized } \\
\text { Values for } \\
\text { MRR }\end{array}$ & $\begin{array}{l}\text { Normalized } \\
\text { Values for } \\
\text { SR }\end{array}$ \\
\hline 1. & 1709 & 2.45 & 0.038062284 & 0 \\
\hline 2. & 2014.86 & 1.415 & 0.610137473 & 0.56557377 \\
\hline 3. & 1970.09 & 1.624 & 0.526400449 & 0.45136612 \\
\hline 4. & 1916.85 & 2.2 & 0.426821285 & 0.136612022 \\
\hline 5. & 2182.26 & 0.788 & 0.923239502 & 0.908196721 \\
\hline 6. & 1997.84 & 1.609 & 0.578303563 & 0.459562842 \\
\hline 7. & 1688.65 & 2.109 & 0 & 0.186338798 \\
\hline 8. & 1997.84 & 1.52 & 0.578303563 & 0.508196721 \\
\hline 9. & 2085.98 & 1.201 & 0.743159076 & 0.682513661 \\
\hline 10. & 2149.19 & 0.801 & 0.861385953 & 0.901092896 \\
\hline 11. & 1896.34 & 2.1 & 0.38845974 & 0.191256831 \\
\hline 12. & 1746.88 & 1.905 & 0.108912373 & 0.297814208 \\
\hline 13. & 1943.11 & 1.887 & 0.475937529 & 0.307650273 \\
\hline 14. & 2009.16 & 1.571 & 0.599476293 & 0.480327869 \\
\hline 15. & 1948.44 & 1.53 & 0.485906668 & 0.50273224 \\
\hline 16. & 2003.48 & 1.709 & 0.58885252 & 0.404918033 \\
\hline 17. & 1751.19 & 1.9 & 0.116973721 & 0.300546448 \\
\hline 18. & 2003.48 & 1.566 & 0.58885252 & 0.483060109 \\
\hline 19. & 1842.16 & 1.91 & 0.287122417 & 0.295081967 \\
\hline 20. & 1751.19 & 1.899 & 0.116973721 & 0.301092896 \\
\hline 21. & 2136.25 & 1.211 & 0.837183204 & 0.67704918 \\
\hline 22. & 1891.29 & 1.999 & 0.379014308 & 0.246448087 \\
\hline 23. & 2055.75 & 1.431 & 0.686617413 & 0.556830601 \\
\hline 24. & 1866.4 & 2.013 & 0.332460488 & 0.238797814 \\
\hline 25. & 1842.16 & 1.945 & 0.287122417 & 0.275956284 \\
\hline 26. & 1916.85 & 2.008 & 0.426821285 & 0.241530055 \\
\hline 27. & 1970.09 & 1.5 & 0.526400449 & 0.519125683 \\
\hline 28. & 1800.08 & 1.789 & 0.208416721 & 0.361202186 \\
\hline 29. & 1937.8 & 1.699 & 0.466005798 & 0.410382514 \\
\hline 30. & 2049.81 & 1.707 & 0.675507341 & 0.406010929 \\
\hline 31. & 2009.16 & 1.5 & 0.599476293 & 0.519125683 \\
\hline 32. & 2142.69 & 0.62 & 0.849228467 & 1 \\
\hline 33. & 1866.4 & 1.934 & 0.332460488 & 0.281967213 \\
\hline 34. & 1916.84 & 2.309 & 0.426802581 & 0.07704918 \\
\hline 35. & 2014.86 & 1.597 & 0.610137473 & 0.466120219 \\
\hline 36. & 2162.3 & 0.8 & 0.885906668 & 0.901639344 \\
\hline 37. & 1800.08 & 1.9 & 0.208416721 & 0.300546448 \\
\hline 38. & 2020.6 & 1.704 & 0.620873469 & 0.407650273 \\
\hline 39. & 1768.66 & 2.102 & 0.149649303 & 0.190163934 \\
\hline 40. & 1842.16 & 2.345 & 0.287122417 & 0.057377049 \\
\hline 41. & 2020.6 & 1.64 & 0.620873469 & 0.442622951 \\
\hline 42. & 2079.86 & 1.634 & 0.731712335 & 0.445901639 \\
\hline 43. & 2162.3 & 0.881 & 0.885906668 & 0.857377049 \\
\hline 44. & 1970.09 & 1.539 & 0.526400449 & 0.497814208 \\
\hline 45. & 1922.04 & 1.997 & 0.43652857 & 0.247540984 \\
\hline 46. & 2223.3 & 0.8 & 1 & 0.901639344 \\
\hline
\end{tabular}


Table 7: Grey Relational Coefficient for Each Performance Co-efficient

\begin{tabular}{|c|c|c|c|c|}
\hline Sl. No. & Material Removal Rate & Surface Roughness & $\begin{array}{c}\text { GRC } \\
\text { Values for MRR }\end{array}$ & GRC Values for SR \\
\hline 1. & 1709 & 2.45 & 0.92926045 & 1 \\
\hline 2. & 2014.86 & 1.415 & 0.450394669 & 0.469230769 \\
\hline 3. & 1970.09 & 1.624 & 0.487139304 & 0.525560023 \\
\hline 4. & 1916.85 & 2.2 & 0.539478331 & 0.785407725 \\
\hline 5. & 2182.26 & 0.788 & 0.351311216 & 0.355064028 \\
\hline 6. & 1997.84 & 1.609 & 0.463691318 & 0.521070615 \\
\hline 7. & 1688.65 & 2.109 & 1 & 0.728503185 \\
\hline 8. & 1997.84 & 1.52 & 0.463691318 & 0.495934959 \\
\hline 9. & 2085.98 & 1.201 & 0.402201142 & 0.422828096 \\
\hline 10. & 2149.19 & 0.801 & 0.367272777 & 0.356864275 \\
\hline 11. & 1896.34 & 2.1 & 0.562771702 & 0.723320158 \\
\hline 12. & 1746.88 & 1.905 & 0.821136214 & 0.626712329 \\
\hline 13. & 1943.11 & 1.887 & 0.512327875 & 0.619079838 \\
\hline 14. & 2009.16 & 1.571 & 0.454761966 & 0.510033445 \\
\hline 15. & 1948.44 & 1.53 & 0.507147397 & 0.498637602 \\
\hline 16. & 2003.48 & 1.709 & 0.459199011 & 0.552536232 \\
\hline 17. & 1751.19 & 1.9 & 0.810407288 & 0.624573379 \\
\hline 18. & 2003.48 & 1.566 & 0.459199011 & 0.508615898 \\
\hline 19. & 1842.16 & 1.91 & 0.635225207 & 0.628865979 \\
\hline 20. & 1751.19 & 1.899 & 0.810407288 & 0.62414734 \\
\hline 21. & 2136.25 & 1.211 & 0.373920341 & 0.424791086 \\
\hline 22. & 1891.29 & 1.999 & 0.568818955 & 0.669838946 \\
\hline 23. & 2055.75 & 1.431 & 0.421365804 & 0.47311272 \\
\hline 24. & 1866.4 & 2.013 & 0.600629107 & 0.676775148 \\
\hline 25. & 1842.16 & 1.945 & 0.635225207 & 0.644366197 \\
\hline 26. & 1916.85 & 2.008 & 0.539478331 & 0.674281503 \\
\hline 27. & 1970.09 & 1.5 & 0.487139304 & 0.490616622 \\
\hline 28. & 1800.08 & 1.789 & 0.705799263 & 0.580583756 \\
\hline 29. & 1937.8 & 1.699 & 0.517595237 & 0.549219688 \\
\hline 30. & 2049.81 & 1.707 & 0.425348258 & 0.551869723 \\
\hline 31. & 2009.16 & 1.5 & 0.454761966 & 0.490616622 \\
\hline 32. & 2142.69 & 0.62 & 0.37058216 & 0.333333333 \\
\hline 33. & 1866.4 & 1.934 & 0.600629107 & 0.639412998 \\
\hline 34. & 1916.84 & 2.309 & 0.539489218 & 0.866477273 \\
\hline 35. & 2014.86 & 1.597 & 0.450394669 & 0.517533937 \\
\hline 36. & 2162.3 & 0.8 & 0.360774655 & 0.356725146 \\
\hline 37. & 1800.08 & 1.9 & 0.705799263 & 0.624573379 \\
\hline 38. & 2020.6 & 1.704 & 0.446080681 & 0.550872968 \\
\hline 39. & 1768.66 & 2.102 & 0.769646019 & 0.724465558 \\
\hline 40. & 1842.16 & 2.345 & 0.635225207 & 0.897058824 \\
\hline 41. & 2020.6 & 1.64 & 0.446080681 & 0.530434783 \\
\hline 42. & 2079.86 & 1.634 & 0.40593894 & 0.528596187 \\
\hline 43. & 2162.3 & 0.881 & 0.360774655 & 0.368357488 \\
\hline 44. & 1970.09 & 1.539 & 0.487139304 & 0.50109529 \\
\hline 45. & 1922.04 & 1.997 & 0.533886542 & 0.668859649 \\
\hline 46. & 2223.3 & 0.8 & 0.333333333 & 0.356725146 \\
\hline
\end{tabular}


Multi-Objective Optimization Of Awjm Of Lead Tin Alloy By Gra

Table 8: Influence of Process Parameters of Grey Relational Grade

\begin{tabular}{|c|c|c|c|c|}
\hline Sl. No. & $\begin{array}{c}\text { Material Removal } \\
\text { Rate }\end{array}$ & Surface Roughness & Grade & Order \\
\hline 1. & 1709 & 2.45 & 0.964630225 & 1 \\
\hline 2. & 2014.86 & 1.415 & 0.459812719 & 37 \\
\hline 3. & 1970.09 & 1.624 & 0.506349663 & 22 \\
\hline 4. & 1916.85 & 2.2 & 0.662443028 & 10 \\
\hline 5. & 2182.26 & 0.788 & 0.353187622 & 44 \\
\hline 6. & 1997.84 & 1.609 & 0.492380966 & 27 \\
\hline 7. & 1688.65 & 2.109 & 0.864251592 & 2 \\
\hline 8. & 1997.84 & 1.52 & 0.479813139 & 34 \\
\hline 9. & 2085.98 & 1.201 & 0.412514619 & 39 \\
\hline 10. & 2149.19 & 0.801 & 0.362068526 & 42 \\
\hline 11. & 1896.34 & 2.1 & 0.64304593 & 11 \\
\hline 12. & 1746.88 & 1.905 & 0.723924271 & 5 \\
\hline 13. & 1943.11 & 1.887 & 0.565703856 & 20 \\
\hline 14. & 2009.16 & 1.571 & 0.482397705 & 33 \\
\hline 15. & 1948.44 & 1.53 & 0.502892499 & 24 \\
\hline 16. & 2003.48 & 1.709 & 0.505867621 & 23 \\
\hline 17. & 1751.19 & 1.9 & 0.717490333 & 6 \\
\hline 18. & 2003.48 & 1.566 & 0.483907454 & 32 \\
\hline 19. & 1842.16 & 1.91 & 0.632045593 & 15 \\
\hline 20. & 1751.19 & 1.899 & 0.717277314 & 7 \\
\hline 21. & 2136.25 & 1.211 & 0.399355714 & 40 \\
\hline 22. & 1891.29 & 1.999 & 0.61932895 & 17 \\
\hline 23. & 2055.75 & 1.431 & 0.447239262 & 38 \\
\hline 24. & 1866.4 & 2.013 & 0.638702128 & 14 \\
\hline 25. & 1842.16 & 1.945 & 0.639795702 & 13 \\
\hline 26. & 1916.85 & 2.008 & 0.606879917 & 18 \\
\hline 27. & 1970.09 & 1.5 & 0.488877963 & 28 \\
\hline 28. & 1800.08 & 1.789 & 0.64319151 & 12 \\
\hline 29. & 1937.8 & 1.699 & 0.533407462 & 21 \\
\hline 30. & 2049.81 & 1.707 & 0.48860899 & 29 \\
\hline 31. & 2009.16 & 1.5 & 0.472689294 & 35 \\
\hline 32. & 2142.69 & 0.62 & 0.351957747 & 45 \\
\hline 33. & 1866.4 & 1.934 & 0.620021053 & 16 \\
\hline 34. & 1916.84 & 2.309 & 0.702983246 & 8 \\
\hline 35. & 2014.86 & 1.597 & 0.483964303 & 31 \\
\hline 36. & 2162.3 & 0.8 & 0.358749901 & 43 \\
\hline 37. & 1800.08 & 1.9 & 0.665186321 & 9 \\
\hline 38. & 2020.6 & 1.704 & 0.498476824 & 25 \\
\hline 39. & 1768.66 & 2.102 & 0.747055789 & 4 \\
\hline 40. & 1842.16 & 2.345 & 0.766142015 & 3 \\
\hline 41. & 2020.6 & 1.64 & 0.488257732 & 30 \\
\hline 42. & 2079.86 & 1.634 & 0.467267564 & 36 \\
\hline 43. & 2162.3 & 0.881 & 0.364566071 & 41 \\
\hline 44. & 1970.09 & 1.539 & 0.494117297 & 26 \\
\hline 45. & 1922.04 & 1.997 & 0.601373096 & 19 \\
\hline 46. & 2223.3 & 0.8 & 0.34502924 & 46 \\
\hline
\end{tabular}

Assessment against the experimental grade values and forecasted grade values and percentage deviation between them are depicted in Table 9. 
Table 9: Percentage Deviation between Experimental and Predicted Grade

\begin{tabular}{|c|c|c|c|c|c|}
\hline SI. No. & $\begin{array}{c}\text { Material } \\
\text { Removal Rate }\end{array}$ & Surface Roughness & $\begin{array}{c}\text { Experimental } \\
\text { Grade }\end{array}$ & Predicted Grade & Percentage Deviation \\
\hline 1. & 1709 & 2.45 & 0.964630225 & 0.869708 & 9.840270667 \\
\hline 2. & 2014.86 & 1.415 & 0.459812719 & 0.466349 & 1.421509344 \\
\hline 3. & 1970.09 & 1.624 & 0.506349663 & 0.522838 & 3.256314332 \\
\hline 4. & 1916.85 & 2.2 & 0.662443028 & 0.647952 & 2.187513126 \\
\hline 5. & 2182.26 & 0.788 & 0.353187622 & 0.343554 & 2.727621603 \\
\hline 6. & 1997.84 & 1.609 & 0.492380966 & 0.486789 & 1.135699129 \\
\hline 7. & 1688.65 & 2.109 & 0.864251592 & 0.842123 & 2.560434086 \\
\hline 8. & 1997.84 & 1.52 & 0.479813139 & 0.485218 & 1.126451333 \\
\hline 9. & 2085.98 & 1.201 & 0.412514619 & 0.482760 & 17.02857977 \\
\hline 10. & 2149.19 & 0.801 & 0.362068526 & 0.362490 & 0.116407273 \\
\hline 11. & 1896.34 & 2.1 & 0.64304593 & 0.636974 & 0.944245153 \\
\hline 12. & 1746.88 & 1.905 & 0.723924271 & 0.725658 & 0.23949036 \\
\hline 13. & 1943.11 & 1.887 & 0.565703856 & 0.577971 & 2.168474513 \\
\hline 14. & 2009.16 & 1.571 & 0.482397705 & 0.486789 & 0.910305913 \\
\hline 15. & 1948.44 & 1.53 & 0.502892499 & 0.504358 & 0.291414283 \\
\hline 16. & 2003.48 & 1.709 & 0.505867621 & 0.527682 & 4.312270218 \\
\hline 17. & 1751.19 & 1.9 & 0.717490333 & 0.730501 & 1.813357764 \\
\hline 18. & 2003.48 & 1.566 & 0.483907454 & 0.486789 & 0.595474574 \\
\hline 19. & 1842.16 & 1.91 & 0.632045593 & 0.639304 & 1.148399241 \\
\hline 20. & 1751.19 & 1.899 & 0.717277314 & 0.726114 & 1.231976262 \\
\hline 21. & 2136.25 & 1.211 & 0.399355714 & 0.455176 & 13.97758546 \\
\hline 22. & 1891.29 & 1.999 & 0.61932895 & 0.628813 & 1.53134288 \\
\hline 23. & 2055.75 & 1.431 & 0.447239262 & 0.447209 & 0.006766337 \\
\hline 24. & 1866.4 & 2.013 & 0.638702128 & 0.615980 & 3.557546892 \\
\hline 25. & 1842.16 & 1.945 & 0.639795702 & 0.620367 & 3.036704067 \\
\hline 26. & 1916.85 & 2.008 & 0.606879917 & 0.643564 & 6.044702052 \\
\hline 27. & 1970.09 & 1.5 & 0.488877963 & 0.489606 & 0.148920019 \\
\hline 28. & 1800.08 & 1.789 & 0.64319151 & 0.688105 & 6.982910914 \\
\hline 29. & 1937.8 & 1.699 & 0.533407462 & 0.508542 & 4.661626273 \\
\hline 30. & 2049.81 & 1.707 & 0.48860899 & 0.461505 & 5.54717389 \\
\hline 31. & 2009.16 & 1.5 & 0.472689294 & 0.485285 & 2.664690404 \\
\hline 32. & 2142.69 & 0.62 & 0.351957747 & 0.324415 & 7.825583333 \\
\hline 33. & 1866.4 & 1.934 & 0.620021053 & 0.615524 & 0.725306449 \\
\hline 34. & 1916.84 & 2.309 & 0.702983246 & 0.666888 & 5.134581192 \\
\hline 35. & 2014.86 & 1.597 & 0.483964303 & 0.486789 & 0.583658142 \\
\hline 36. & 2162.3 & 0.8 & 0.358749901 & 0.338711 & 5.585757814 \\
\hline 37. & 1800.08 & 1.9 & 0.665186321 & 0.711362 & 6.941766153 \\
\hline 38. & 2020.6 & 1.704 & 0.498476824 & 0.499514 & 0.20806896 \\
\hline 39. & 1768.66 & 2.102 & 0.747055789 & 0.749438 & 0.318879989 \\
\hline 40. & 1842.16 & 2.345 & 0.766142015 & 0.759574 & 0.857284309 \\
\hline 41. & 2020.6 & 1.64 & 0.488257732 & 0.486789 & 0.300810744 \\
\hline 42. & 2079.86 & 1.634 & 0.467267564 & 0.461961 & 1.135658477 \\
\hline 43. & 2162.3 & 0.881 & 0.364566071 & 0.339166 & 6.967206621 \\
\hline 44. & 1970.09 & 1.539 & 0.494117297 & 0.484763 & 1.893132873 \\
\hline 45. & 1922.04 & 1.997 & 0.601373096 & 0.643108 & 6.939935394 \\
\hline 46. & 2223.3 & 0.8 & 0.34502924 & 0.301157 & 12.71551356 \\
\hline
\end{tabular}

The mathematical model for the experimental data by cutting the Lead Tin alloy using abrasive water jet machine for MRR and SR is developed using linear regression analysis by Minitab software. The developed regression equations are given below.

Material Removal Rate $=-1897+1.0089$ Pressure + 307.6 Flow Rate of Abrasive + 349 Diameter of Orifice +62 Diameter of Focusing Nozzle - 61.02 Stand Off Distance
Surface Roughness $=12.10-0.002798$ Pressure -1.311 Flow Rate of Abrasive 1.33 Diameter of Orifice +0.219 Diameter of Focusing Nozzle +0.2820 Stand Off Distance Grade $=0.6086+0.20656$ Pressure $3400-0.02617$ 
Pressure 3600 - 0.18039 Pressure $3800+0.08445$ Flow Rate of Abrasive 0.40 - 0.03815 Abrasive Flow Rate 0.55 0.04631 Flow Rate of Abrasive $0.70+0.01775$ Orifice Diameter 0.30 - 0.01217 Orifice Diameter 0.33 - 0.00558 Orifice Diameter $0.35+0.00527$ Focusing Nozzle Diameter 0.90 - 0.00570 Focusing Nozzle Diameter $0.99+0.00043$



Figure 5: Comparison of Predicted \&Experimental MRR
Focusing Nozzle Diameter 1.05 - 0.07101 Stand Off Distance $1-0.03959$ Stand Off Distance $2+0.11060$ Stand Off Distance 3

Assessment against forecasted and experimental values of MRR, SR and Grade is depicted in Figure 5, 6 and 7.



Figure 6: Comparison of Predicted \& Experimental SR



Figure 7: Comparison of Predicted Grade and Experimental Grade

\section{Effect of Process Parameters on Grade}

The contour plot showed in figure 8 briefs the anticipated response surface for Grade concerning not only pressure but also the flow rate of the abrasive. It exhibits as $\mathrm{P}$ and $\mathrm{m}_{\mathrm{f}}$ decreases, i.e, once $P$ is 3400 bar and $m_{f}$ is $0.4 \mathrm{~kg} / \mathrm{min}$, the grade obtained is high. Figure 9 illustrates as the stand off distance $\mathrm{s}$ increases and $\mathrm{P}$ decreases, the grade obtained is



Figure 8: Grade vs Pressure and Abrasive Flow Rate high, i.e, if $\mathrm{s}$ is $3 \mathrm{~mm}$ and $\mathrm{P}$ is 3400 bar. Figure 10 illustrates as $d_{o}$ declines and $m_{f}$ declines too i.e, once $d_{o}$ is $0.3 \mathrm{~mm}$ and $\mathrm{m}_{\mathrm{f}}$ is $0.4 \mathrm{~kg} / \mathrm{min}$ the grade obtained is high. Figure 11 shows as the standoff distance increases and flow rate of abrasive decreases obtains high grade, i.e, once $\mathrm{s}$ is $3 \mathrm{~mm}$ and $\mathrm{m}_{\mathrm{f}}$ is $0.4 \mathrm{~kg} / \mathrm{min}$. The residual plots for grade is depicted in Figure 12.



Figure 9: Grade vs Stand off Distance and Pressure






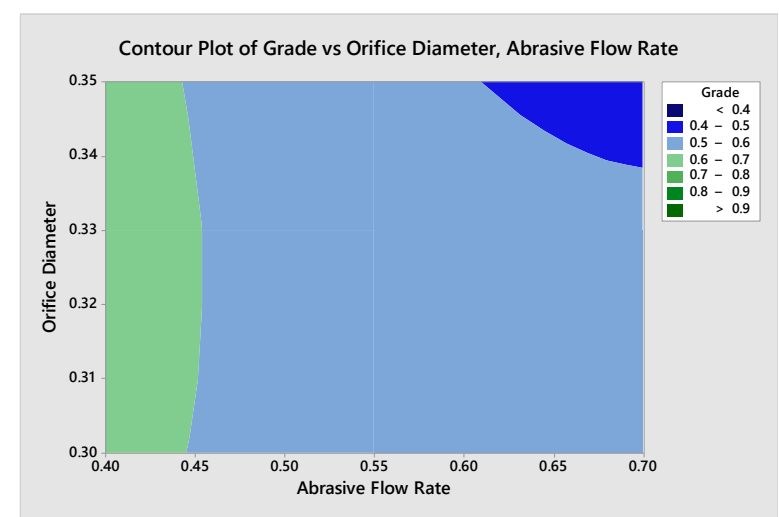

Figure 10: Grade vs AFR and Orifice Diameter



Figure 11: Grade vs Stand Off Distance and AFR

Residual Plots for Grade



Figure 12: Residual Plots for Grade for Lead Tin Alloy

\section{Analysis of Variance}

ANOVA is done in Minitab software. ANOVA is a great analyzing tool to examine that design parameters extensively influence the quality feature. Conventional statistic method acquire single parameter in a single sequence and is noticed that $\mathrm{P}$ is the major factor for making the most of the MRR and least of the SR and next is the $m_{f}$, $\mathrm{s}, \mathrm{d}_{\mathrm{o}}$ and $\mathrm{d}_{\mathrm{f}}$. The increase in pressure end up in greater velocity of water jet that impacts on the material increases the MRR and produces good SR. The contribution of each machining parameters that affect the abrasive water jet machining process is also shown in table 10 and figure 13. From the Grey relational grade plot in figure 14 the optimal design is identified and this design is verified by means of confirmation test.

Table 10: Analysis of Variance for Grade, Using Adjusted SS for Tests

\begin{tabular}{|c|c|c|c|c|c|c|c|}
\hline Source & DF & Seq SS & Adj SS & Adj MS & F & P & Contribution (\%) \\
\hline Pressure & 2 & 0.611775 & 0.611775 & 0.305887 & 313.17 & 0.000 & 67.6300 \\
\hline $\begin{array}{c}\text { Abrasive Flow } \\
\text { Rate }\end{array}$ & 2 & 0.095715 & 0.095715 & 0.047857 & 49.00 & 0.000 & 10.7374 \\
\hline Orifice Diameter & 2 & 0.004956 & 0.004956 & 0.002478 & 2.54 & 0.094 & 0.5559 \\
\hline $\begin{array}{c}\text { Focusing Tube } \\
\text { Diameter }\end{array}$ & 2 & 0.000705 & 0.000705 & 0.000352 & 0.36 & 0.700 & 0.0790 \\
\hline $\begin{array}{c}\text { Stand-off } \\
\text { Distance }\end{array}$ & 2 & 0.169885 & 0.169885 & 0.084943 & 86.97 & 0.000 & 18.0580 \\
\hline Error & 35 & 0.034186 & 0.034186 & 0.000977 & & & 3.8350 \\
\hline Lack of Fit & 31 & 0.034119 & 0.034119 & 0.001101 & 65.56 & 0.000 & \\
\hline Pure Error & 4 & 0.000067 & 0.000067 & 0.000017 & & & 100 \\
\hline \multicolumn{7}{|c|}{$\mathrm{S}=0.0312529 \mathrm{R}-\mathrm{Sq}=96.16 \% \mathrm{R}-\mathrm{Sq}(\mathrm{adj})=95.07 \% \mathrm{R}-\mathrm{sq}(\mathrm{pred})=93.32 \%$} \\
\hline
\end{tabular}






Figure 13: Contribution of Each Machining Parameters

\section{Confirmation Test}

Confirmation test is utilized for proving the accurateness of the built up model after identifying the optimal design. The investigational result which is having the high grade value utilizing the preliminary arrangement of the parameters involved in cutting is assessed against the optimal one which has got from the mean effects plot shown in figure 10. Then the experiment is done with the new optimal design for MRR and SR and from table 11, it is observed that the MRR increases from $2142.69 \mathrm{~mm}^{3} / \mathrm{min}$ to $2144.34 \mathrm{~mm}^{3} / \mathrm{min}$ and SR decreases from $0.620 \mu \mathrm{m}$ to $0.618 \mu \mathrm{m}$ in the optimal combination of cutting parameters. By this test, it is understood that quality characteristics of cutting of Lead Tin alloy using abrasive water jet machine could be improved to a great extent.

Table 11: Result of Confirmation Tests

\begin{tabular}{|c|c|c|c|c|c|c|c|}
\hline \multirow{2}{*}{$\begin{array}{c}\text { Desig } \\
\mathbf{n}\end{array}$} & \multicolumn{6}{|c|}{ Process Parameters } & \multicolumn{2}{c|}{ Output } \\
\cline { 2 - 8 } & $\mathbf{P}$ & $\begin{array}{c}\mathbf{m}_{\mathbf{f}} \\
\mathbf{( B a}\end{array}$ & $\begin{array}{c}\mathbf{d}_{\mathbf{o}} \\
\mathbf{( \mathbf { m }} / \mathbf{m}\end{array}$ & $\begin{array}{c}\mathbf{d}_{\mathbf{f}} \\
\mathbf{( m}\end{array}$ & $\begin{array}{c}\mathbf{S} \\
\mathbf{( m}\end{array}$ & $\begin{array}{c}\text { MRR } \\
\left(\mathbf{m m}^{\mathbf{3}} /\right.\end{array}$ & $\begin{array}{c}\mathbf{S R} \\
(\boldsymbol{\mu}\end{array}$ \\
\hline Initia & 380 & 0.7 & 0.3 & 0.9 & 2 & 2142.69 & 0.6 \\
\hline Opti & 380 & 0.7 & 0.3 & 0.9 & 1 & 2144.34 & 0.6 \\
\hline
\end{tabular}

\section{CONCLUSION}

In this paper, optimization of abrasive water jet machining process of Lead Tin alloy by grey relational analysis has been presented. Using linear regression analysis a mathematical model is developed by Minitab software. Using ANOVA the significant parameter that affects the quality characteristics has been found out and from the main effects plot the optimal design has been identified with pressure $3800 \mathrm{bar}$, flow rate of abrasive $0.7 \mathrm{~kg} / \mathrm{min}$, diameter of orifice $0.35 \mathrm{~mm}$, diameter of focusing nozzle is of $0.9 \mathrm{~mm}$ and $1 \mathrm{~mm}$ of standoff distance as the best combination which provides the maximum MRR and minimum SR. By experimentation and ANOVA the optimal design is verified.

\section{REFERENCES}

1. Momber A.W., Kovacevic R., (1992), Principles of Abrasive Water Jet Machining. Springer Verlag Limited, London.

2. Hashish M., (1989), A Model for Abrasive Water Jet [AWJ] Machining. Transactions of ASME Journal of Engineering Materials and Technology, Vol. III: pp. 154162.

3. R. Kovacevic, M. Fang, "Modeling of the Influence of the Abrasive Water Jet Cutting Parameters on the Depth



Figure 14: Grey Relational Grade Plot for Lead Tin Alloy in ANOVA

of Cut Based on Fuzzy Rules", International Journal of Machine Tools Manufacture, Vol. 34, No.1, pp.55-72, 1994.

4. P. Asokan, R. Ravi Kumar, R. Jeyapaul and M. Santhi, (2008), "Development of Multi-Objective optimization Model for Electrochemical Machining Process". International Journal of Advanced Manufacturing Technology, (2008), Vol. 39, pp. 55-63.

5. D. Chakradhar and A. Venu Gopal, (2011), "MultiObjective Optimization of Electrochemical Machining of EN31 Steel by Grey Relational Analysis", International Journal of Modeling and Optimization (2011), Vol. 1, No 2, pp 113-117.

6. C.L. Lin, (2004), "Use of the Taguchi Method and Grey Relational Analysis to Optimize Turning Operations with Multiple Performance Characteristics", Materials and Manufacturing Processes (2004), Vol. 19, No. 2, pp. 209-220.

7. N. Natarajan and R.M. Arunachalam, (2011), "Optimization of Micro-EDM with Multiple Performance Characteristics Using Taguchi Method and Grey Relational Analysis", Journal of Scientific and Industrial Research, (2011), Vol. 70, pp. 500-505

8. C.L. Lin, J. L. Lin and T. C. Ko, (2002), "Optimization of the EDM Process Based on the Orthogonal Array with Fuzzy Logic and Grey Relational Analysis Method", International Journal of Advanced Manufacturing Technology, (2002), Vol. 19, pp. 271-277.

9. Chithirai Pon Selvan M, N. Mohana Sundara Raju., "Modeling and Analysis of Depth of Cut in Abrasive Water Jet Cutting of Titanium", International Journal of Mechanical Engineering and Technology, Vol. 2, Issue 2, pp. 39-46, 2011.

10. Chithirai Pon Selvan M. and Mohanasundara Raju N, (2012), Influence of Abrasive Water Jet Cutting Conditions on Depth of Cut of Mild Steel, International Journal of Design and Manufacturing Technology, ISSN: 0976 - 7002, Vol. 3, Issue 1, January- December (2012), pp. 48-57.

11. Chithirai Pon Selvan M. and Mohanasundara Raju N, (2012), Selection of Process Parameters in Abrasive Water Jet Cutting of Copper, International Journal of Advanced Engineering Sciences and Technologies, Vol. 7, Issue 2, pp. $254-257$.

12. M. A. Azmira, A. K. Ahsanb, (2009), A Study of Abrasive Water Jet Machining Process on Glass/Epoxy Composite Laminate, Journal of Materials Processing Technology, Vol. 209, (2009), pp. 6168-6173 